\title{
Educational program strategy in rising the awareness for adverse drug reactions reporting and improved pharmacovigilance practice in the Republic of North Macedonia
}

\author{
Iskra P. Sadikarijo ${ }^{1}$, Marijana Danevska ${ }^{1}$, Vera Georgieva ${ }^{1}$, Zorica Naumovska ${ }^{2}$ \\ ${ }^{1}$ Agency for drugs and medical devices, Blvd. SS. Cyril and Methodius 54, 1000 Skopje, N. Macedonia \\ ${ }^{2}$ Faculty of Pharmacy, Ss. Cyril and Methodius University, Mother Theresa 47, 1000 Skopje, N. Macedonia
}

\section{Introduction}

Pharmacovigilance (PV) is defined with the World Health Organization (WHO) definition as "the science and activities relating to the detection, assessment, understanding and prevention of adverse effects or any other medicine-related problems". Having undergone rapid growth over the past two decades, today, PV includes many other disciplines in the research and development of drugs. This growth has come resulted in heightened awareness and interest in the medical community about the roles that PV plays (Beninger, 2018). No country has a perfect pharmacovigilance system and we are facing underreporting globally. Well established PV system in the national level is inevitable for better patient care, improved public health, increased drugs safety and evaluation of risk/benefit ratio. Additionally, it is very important to be a part of global pharmacovigilance system in order to be upto-date with all important issues concerning drugs utilized in everyday practice by the HCP and patients (Martin et al., 2018). Since 2015 National PV center in the Republic of North Macedonia is undertaken by the National competent authority -
MALMED. This center works in accordance with the National regulative for pharmacovigilance, which is not fully harmonized with EU legislative Regulative (EU) 1235/2010, which was adopted in 2012, and since then it is continuously improved. Since 2002 Macedonia is a full member in the Uppsala monitoring center. Although the National center for pharmacovigilance exists for almost twenty years, the awareness for reporting adverse drug reactions (ADR) by the healthcare professionals and patients is still low (GVP, EMA). Since 2017 MALMED has established easy access to ADR reporting form for health care professionals (HCPs) and patients. Also state of art software system was grounded which enables easy and safe transfer of all reports in E2B R3 format according to EU legislative among all stakeholders, Agency, Marketing authorization holders (MAHs), Uppsala monitoring system (UMC) and Ministry of health.

This study aimed to evaluate the efficacy of implemented measures for improvement of knowledge and awareness of adverse drug reactions (ADRs) reporting among health care professionals (HCPs) including hospital pharmacists.

\footnotetext{
* iskra.sadikarijo@malmed.gov.mk
} 


\section{Materials and Methods}

Training program as educational workshops for pharmacovigilance was organized and conducted by MALMED among 500 HCPs in twelve different cities. A community pharmacists were included from different regions from RN Macedonia. Subsequently non - interventional, questionnaire based study was enrolled in order to evaluate the improvement of knowledge and attitude toward ADR reporting and pharmacovigilance system. The official data were obtained from the Macedonian agency of drugs and medical devices (MALMED).

\section{Results and Discussion}

High percentage of awareness and knowledge for importance of ADR reporting was confirmed (over $95 \%$ of the participants), even before the implementation of educational program. Good theoretical understanding for pharmacovigilance was observed among HCPs and a positive attitude towards ADR reporting was presented by all participants. It was considered that ADRs reporting is contributing to utilization of safer drugs in clinical practice which is the major benefit from implementation of good PV practice. ADR reporting was associated with improvement of the quality of patient care and HCPs considered that the new information for the drugs obtained from good PV practice will contribute to rational use of drugs. All this will add to increased trust in the health service and help in improving the relationship between patients and health care professionals (HCPs).

Lack of sufficient information for the importance of ADR reporting, lack of knowledge whom to report or inappropriate fear of medical and legal issues were noticed as leading factors for underreporting. Additionally, the lack of time was addressed as the main limitation factor for implementation of appropriate PV practice, as well as by the uncertainty for association between occurred adverse drug event (ADE) and utilization of certain drug. The hospital pharmacists were nominated as main stakeholders in the improvement process for ADR reporting and have undertaken the responsibility for reporting toward Macedonian regulatory MALMED.

Conducted educational workshops with HCPs, improvement of theoretical knowledge alongside with practical skills for process of ADR reporting, development of website application by MALMED for easy access to reporting form for HCPs and patients, dedicated participation of all stakeholders resulted in huge progress in pharmacovigilance system and practice in the RN Macedonia. The number of reported ADRs in period of one year increased ten folds, 48 reports in December 2017 vs 505 in December 2018. In 2019 the number of submitted ADRs was over 300. This well established system is a key factor in improvement of PV practice and easy access for all HCPs and patients.

\section{Conclusion}

Implementation of appropriate strategy consisting of PV educational program with workshops alongside with contemporary software infrastructure was confirmed as a successful approach for improvement of ADR reporting in everyday clinical practices. Implemented educational and training program among HCPs with pivotal role of hospital pharmacist encouraged them for active participation in ADR reporting on the national level.

\section{References}

Beninger, P., 2018. Pharmacovigilance: An overview. Clin. Ther. 40(12), 1991-2004.

Directive 2010/84/EU of the European Parliament and of the Council of 15 December 2010.

Law for medicinal products and medical devices Act. Official Gazette No. 106/07 (Available at: https://malmed.gov.mk).

Martin, L.G., Hanssens, Y., Paudyal, V., 2018. Overview of this issue: pharmacovigilance, what is new?. Int. J. Clin. Pharm. 40(4), 737-739.

Module VIII Addendum I - Requirements and recommendations for the submission of information on non-interventional post-authorisation safety studies (Rev 2).

Regulation (EU) 1235/2010 of the European Parliament and of the Council of 15 December 2010.

Maced. Pharm. Bull. 66 (Suppl 1) 93 - 94 (2020) 